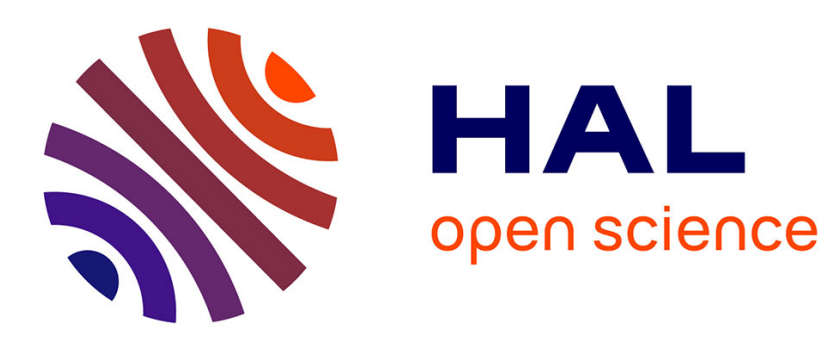

\title{
Vers des systèmes de mesure des performances sociétales : l'apport des conventions
}

\author{
Marc Bollecker, Pierre Mathieu
}

\section{To cite this version:}

Marc Bollecker, Pierre Mathieu. Vers des systèmes de mesure des performances sociétales: l'apport des conventions. Revue Française de Gestion, 2008, 180, pp.89-102. halshs-00769061

\section{HAL Id: halshs-00769061 https://shs.hal.science/halshs-00769061}

Submitted on 27 Dec 2012

HAL is a multi-disciplinary open access archive for the deposit and dissemination of scientific research documents, whether they are published or not. The documents may come from teaching and research institutions in France or abroad, or from public or private research centers.
L'archive ouverte pluridisciplinaire HAL, est destinée au dépôt et à la diffusion de documents scientifiques de niveau recherche, publiés ou non, émanant des établissements d'enseignement et de recherche français ou étrangers, des laboratoires publics ou privés. 


\title{
Vers des sytèmes de mesure des performances sociétales : l'apport des conventions
}

\author{
Marc Bollecker et Pierre Mathieu \\ Maîtres de conférences en Sciences de Gestion \\ Centre d'Analyse et de Recherche en Gestion des Organisations \\ Université de Haute Alsace
}

Correspondance :

Marc Bollecker

34 rue de Champlitte

68790 Morschwiller-le-bas

m.bollecker@uha.fr 


\section{Vers des systèmes de mesure des performances sociétales: l'apport des conventions}

\section{Résumé}

L'intérêt croissant accordé par les praticiens et les chercheurs à la responsabilité sociale des entreprises les conduit, depuis quelques années, à proposer des évolutions majeures dans les systèmes de mesure de la performance. Après avoir cerné les sources d'émergence du concept de responsabilité sociale, au travers de la théorie néo-institutionnelle, notre contribution montre que les évolutions de ces systèmes sont susceptibles de se heurter à des difficultés, notamment humaines. La théorie des conventions constitue un cadre conceptuel permettant de comprendre les origines de ces problèmes en appréhendant l'ensemble des outils de mesure de la performance comme des conventions d'effort. Une analyse de la dynamique de ces dernières permet alors de proposer des modalités incitant les individus à adopter des comportements compatibles avec des systèmes de mesure de la performance sociétale. 


\section{Introduction}

Depuis quelques années fleurissent, dans le domaine des Sciences de Gestion, des problématiques renouvelées de la performance et de son contrôle, articulées autour du concept émergent de Responsabilité Sociétale. Cette évolution des réflexions est, bien entendu, liée aux différentes transformations des environnements socio-économiques des entreprises qui conduisent à l'élargissement de leur responsabilité à l'ensemble des membres du corps social vis-à-vis desquels leurs actions ont un effet.

Partant du constat selon lequel l'atteinte d'objectifs complexes multidimensionnels nécessite un renouvellement profond des systèmes de mesure des performances, notre objectif est de proposer une analyse des conditions de réussite de cette évolution. La première partie de notre contribution consiste en une présentation succincte de l'évolution des systèmes de mesure des performances en relation avec le développement du concept de responsabilité sociétale. Le cadre analytique de la théorie néo-institutionnelle permet, tout d'abord, de caractériser les différentes évolutions environnementales à l'origine des changements d'organisations. Les principales logiques de renouvellement des systèmes de mesure de la performance puis leurs difficultés d'utilisation sont ensuite abordées. Dans la seconde partie, l'appareillage analytique de la théorie des conventions est mobilisé afin d'observer le rôle des systèmes de mesures des performances sur les comportements individuels et collectifs via la notion de « conventions d'effort ». Nous soulignons ainsi la nécessité d'analyser le renouvellement de ces systèmes comme une évolution majeure des conventions d'efforts appliquées au sein de l'organisation. L'enjeu consiste dès lors à concevoir le système de mesure à l'aune des comportements souhaités. 


\section{Responsabilité sociétale et systèmes de mesure de la performance}

De très nombreuses contributions récentes abordent le thème de la Responsabilité Sociétale des Entreprises et ses multiples implications gestionnaires. Ce concept, bien que pouvant faire l'objet de différentes définitions, s'entend très généralement comme «l'intégration volontaire des préoccupations sociales et écologiques des entreprises à leurs activités commerciales et leurs relations avec leurs parties prenantes.»(Commission des Communautés Européennes, 2001). Il s'agit donc d'élargir le champ naturel de la performance de l'entreprise afin de proposer, dans une perspective de Développement Durable, une vision tridimensionnelle de la responsabilité des organisations (Gélinier, 2004): économique, environnementale et sociale. Une telle évolution soulève des interrogations gestionnaires considérables dès lors que l'on postule, avec Igalens (2003b, p. 55), que «la responsabilité sociale de l'entreprise n'est pas une mode, c'est un véritable changement de paradigme car elle comporte une nouvelle manière de penser l'entreprise, sa légitimité et sa relation aux autres composantes de la société ». Le développement de ce paradigme résulte, pour l'essentiel, d'une nette évolution de l'environnement des entreprises (1.1). Beaucoup d'organisations, afin de s'adapter à un contexte renouvelé, s'engagent ainsi dans des modifications substantielles de leurs systèmes de mesure des performances (1.2.) dont l'utilisation soulève certaines difficultés méthodologiques importantes (1.3.).

\subsection{Le contexte Institutionnel de la Responsabilité Sociétale}

Depuis une dizaine d'années, de nombreux questionnements sur la place de l'entreprise au sein de nos sociétés se sont fait jour et se sont diffusées largement à l'ensemble du corps 
social. Il ne s'agit cependant pas d'une interrogation nouvelle puisque des préoccupations sociétales ont régulièrement fait irruption dans les réflexions sur les organisations depuis le milieu du siècle dernier ${ }^{1}$. Très succinctement, il est possible d'identifier deux questionnements visant à concilier le fondement du système capitaliste et la recherche du bien être collectif maximal. D’une part, la propriété privée des moyens de production confère aux propriétaires du capital le pouvoir de décider librement de la gestion de leurs patrimoines et donc fondamentalement celui de la genèse de l'activité économique. Comment, dès lors, préserver les intérêts des différents stakeholders dont le pouvoir apparaît nécessairement de second rang par rapport à celui des actionnaires? D'autre part, la maximisation des objectifs individuels produit nécessairement des externalités puisqu'elle implique des choix relatifs à l'utilisation de ressources rares et qu'elle ne s'obtient généralement qu'au détriment des concurrents et de certaines prties prenantes. Comment, dès lors, concilier les décisions individuelles des entreprises avec l'intérêt collectif ? Ces interrogations théoriques, combinées à une évolution très sensible de l'environnement sont à l'origine de réflexions profondes sur les buts fondamentaux et les modèles d'organisation des entreprises. Le cadre conceptuel proposé par les sociologues néo-institutionnels (Dimaggio et Powell, 1991 ; Greenwood et Hinings, 1996) permet d'identifier et d'analyser trois mouvements complémentaires.

L'évolution du cadre réglementaire et juridique est, tout d'abord, à l'origine d'un changement des organisations du type «isomorphisme coercitif ». C'est l'évolution des contraintes qui justifie une stratégie d'adaptation de l'entreprise et, in fine, un changement d'organisation et une évolution des systèmes de mesure des performances. En se limitant à la France, on peut analyser les décisions en matière, par exemple, d'intéressement et de participation (initiées durant les années 1950), de formation professionnelle et de bilan social (années 1970),

\footnotetext{
1 Le développement du concept de «Corporate Social Responsability » date des années 1950. Pour une analyse bibliographique approfondie voir Carrol (1999) et Wood (1991).
} 
d'expression et de représentation des salariés (début des années 1980) puis, plus récemment, d'information des stakeholders comme autant de tentatives visant à renforcer le pouvoir d'action ou de réaction des différents membres du corps social vis-à-vis des entreprises. Plus récemment, d'autres formes de contraintes ont émané de parties prenantes différentes (salariés, consommateurs, médias, ONG, communauté scientifique, système judiciaire, etc.). Elles se sont, en particulier, développées suite à une forte réaction du corps social à des décisions de gestion très fortement symboliques (par exemple fermetures des usines Renault à Wilwoorde et Metaleurope à Noyelles-Godault, affaires Danone, Nike, Mark's and Spencer, etc.). Les « contrepouvoirs » imaginés prennent alors des formes très variées : boycottage des produits ou d'une marque, manifestations, dénigrements, perturbations d'Assemblées Générales, etc. Au-delà des considérations sociales, ces réactions des stakeholders se sont également nourries de la démonstration scientifique des limites de la croissance démographique et économique en termes de gestion des ressources environnementales. La prise de conscience du pouvoir de nuisance de l'homme au travers, essentiellement, du développement de ses activités économiques, constitue en effet une évolution majeure dans les perceptions individuelles et collectives des liens entre sphères privées et sphère publique.

Une seconde source d'évolution des entreprises s'inscrit dans la logique de «l'isomorphisme normatif ». C'est le développement de normes et de standards par différentes institutions du corps social qui amène les gestionnaires à modifier leurs organisations. Comme l'expliquent Chauveau et Rosé (2003), le pouvoir coercitif des institutions publiques, en particulier au niveau international, reste en effet limité. Différentes «institutions » se constituent dès lors autour du champ conceptuel du Développement Durable afin d'élaborer plusieurs standards. Les initiatives les plus marquantes sont la publication du rapport Brundtland (1987), l'adoption du l'agenda 21 lors du sommet de Rio en 1992, la « Global Reporting Initiative» en 
1997, le «Global Compact » proposé par le Secrétaire Général de l'ONU en 1998. Dans le même temps, de nombreuses «normes » destinées aux entreprises sont développées dans les champs de la responsabilité environnementale et de la responsabilité sociale (ISO 14001, SA 8000, etc.). Enfin, ce développement du cadre normatif s'accompagne de nombreuses réflexions et tentatives d'amélioration des systèmes de « rating », principalement à des fins d'information des gestionnaires ou des investisseurs.

Enfin, la période la plus récente est marquée par le développement d'un troisième mécanisme d'évolution du type «isomorphisme mimétique». Ce sont les initiatives de certaines entreprises novatrices qui conduisent leurs concurrents ou partenaires à s'engager dans la même voie. Naturellement, ce mimétisme est favorisé par le développement de réseaux de responsables d'entreprises et de gestionnaires par exemple l'Observatoire sur le Responsabilité Sociétale des Entreprises (ORSE) en France et le World Business Council for Sustainable Development (WBCSD) au niveau international. Ce phénomène est d'autant plus marquant que les chercheurs soulignent la très grande difficulté à établir une relation ferme entre l'adoption de pratiques «socialement responsables » et la performance économique et financière.

Le changement de paradigme souligné par Igalens (2003b) implique donc, parmi d'autres effets, une interrogation approfondie sur la finalité, la nature et les caractéristiques des systèmes de mesure des performances susceptibles d'intégrer concomitamment des considérations économiques, sociales et environnementales. Au-delà de ce mécanisme, Il convient également de s'interroger, plus fondamentalement, sur les conditions nécessaires pour qu'une évolution des systèmes de mesure des performances se traduise réellement par un renouvellement des comportements individuels et collectifs au sein des organisations.

\subsection{L'évolution des systèmes de mesure de la performance}


Les systèmes de mesure de la performance ont fait l'objet, depuis le début des années 1990, de nombreux travaux portant sur leur adaptation aux stratégies organisationnelles et/ou à l'environnement économique (Lorino, 1991; Malo et Mathé 2000 ; Mavrinac et Siesfeld 1998 ; Bollecker, 2001 ; Mathieu, 2000). En effet, les évolutions majeures survenues dans la stratégie depuis plusieurs décennies -notamment la rénovation des fondements de la compétitivité par la qualité, les services périphériques et l'innovation- ainsi que les questionnements récents relatifs aux modes de gouvernance des entreprises (Perez, 2003) ont posé la question de la transformation des modalités d'évaluation de la performance.

Dans une approche d'inspiration « contractualiste », la théorie de la gouvernance partenariale -élaborée à la suite de l'article novateur de Charreaux et Desbrières (1998) - constitue, par exemple, un cadre d'analyse très riche des objectifs multidimensionnels des organisations et des enjeux en termes de modes de gouvernance. En rupture avec la Gouvernance d'entreprise « orientée actionnaires », il s'agit en effet d'analyser la valeur partenariale de l'organisation et d'étudier les modes de répartition de la rente entre les différentes parties prenantes.

Dans une perspective plus opérationnelle, certaines contributions se fondent sur la valorisation financière de la performance sociétale grâce à des systèmes comptables. A partir de données monétaires sur les coûts supportés par les entreprises et d'informations sur le coût des dommages imposés à la société (Gray, 1992), ces systèmes contribuent à informer la société civile des pratiques environnementales - au sens écologique - en vigueur dans les organisations. De la même manière, la comptabilité des ressources humaines permet de mesurer l'impact social des décisions économiques d'une entité sur son environnement (Capron, 1995).

De nombreux spécialistes ont également proposé de greffer à la traditionnelle représentation de l'organisation par les systèmes comptables, une représentation qualitative qui s'est traduite 
par l'intégration d'informations non financières (Chiapello et Delmond, 1994 ; Boisvert, 1991). Une modélisation de la performance a ainsi été proposée dans différents travaux, notamment dans ceux de Kaplan et Norton (1998), dans le but de mieux cerner le fonctionnement des processus internes, la capacité à l'apprentissage organisationnel, la satisfaction des clients et des actionnaires dans le «balanced scorecard».

Des auteurs comme Edvinsson et Malone (1999) vont plus loin dans la démarche au travers du «Skandia Navigator». Le principe de ce tableau de bord est de mesurer la performance sociétale par le capital humain pour fournir des éléments de contrôle managérial et des informations à destination de toutes les parties prenantes, en complément des différents critères retenus par Kaplan et Norton. Bieker (2002), Hockerts (2001) ou Kaplan et Norton (2001) eux-mêmes proposent une approche différente de la mesure de la performance sociétale. L'idée est de compléter l'approche du balanced scorecard en y intégrant un axe «société » pour représenter la dimension sociétale dans un «sustainability balanced scorecard». Des indicateurs de «comportement citoyen » peuvent ainsi faire l'objet d'une mesure de la performance.

Le modèle d'excellence de l'European Foundation for Quality Management (EFQM) ou le modèle de la performance du Baldrige Quality Award (BQA) vont dans le même sens (Oger et Pratt, 2002). Ces modèles, reconnus en Europe pour le premier, aux Etats-Unis pour le second, constituent des outils de mesure de la performance sur des critères très vastes notamment de performance sociétale.

En France, le Centre des Jeunes Dirigeants et Acteurs de l'Economie Sociale (CJDES) propose un outil qui accorde une place encore plus importante que les modèles précédents à la performance vis-à-vis des parties prenantes (Capron, 2003). Le bilan sociétal intègre en effet de nombreuses informations dans différentes thématiques (activité et relations clients, gestion 
économique, anticipation, prospective, innovation, production et organisation du travail, ressources humaines, acteurs internes, citoyens de l'entreprise, environnement humain, social et institutionnel, environnement biophysique, finalités, valeurs et éthique) qui complètent largement celles intégrées dans les systèmes de mesure de la performance plus classiques. L'intérêt du bilan sociétal est surtout de mettre en valeur les antagonismes pouvant apparaitre à partir des demandes contradictoires des partenaires de l'organisation et de favoriser les négociations, les discussions entre les différentes catégories d'acteurs.

Le développement de ces outils traduit donc une évolution de la mesure de la performance vers une prise en compte de la responsabilité sociétale des entreprises. Une telle évolution est toutefois à relativiser dans la mesure où elle apparaît essentiellement au travers de contributions conceptuelles. Les travaux empiriques portant sur les indicateurs de performance ou sur les tableaux de bord se font encore trop rares.

\subsection{Les difficultés liées à l'utilisation des systèmes de mesure de la performance}

Cet engouement est également à nuancer dans la mesure où ces outils ne sont pas exempts de problèmes, notamment humains. De nombreux travaux, menés surtout en contrôle de gestion à partir du début des années 1950, montrent en effet que les systèmes de mesure de la performance créent des tensions chez les utilisateurs, conduisent à un climat de défiance voire de dénonciation et à des luttes de territoires (Argyris, 1952) ainsi qu'à des marges de manœuvre budgétaires (Merchant, 1985). L'évaluation liée à ces systèmes présente des difficultés dans la mesure où les individus ne maîtrisent pas tous les paramètres qui influencent leurs performances. Certains styles budgétaires peuvent même conduire les responsables à falsifier les données (Hopwood, 1972). 
Ces constats nous conduisent à considérer que des réflexions portant sur la dimension technique des systèmes de mesure de la performance sociétale doivent s'accompagner de recherches portant sur leur dimension humaine. Plus précisément, il semble nécessaire de s'attarder sur les mécanismes incitant les individus à agir dans le sens de la performance sociétale que mesurent les outils évoqués précédemment. Cette problématique est souvent traitée dans une perspective contractualiste. Par exemple, Kaplan et Norton (2001) estiment que la récompense financière constitue une puissante motivation pour inciter les individus à atteindre les mesures du balanced scorecard.

Ces méthodes incitatives ne se distinguent pas ou peu de celles à l'origine des difficultés, évoquées précédemment et liées à l'utilisation des systèmes de mesure de la performance. Elles présentent la caractéristique de conserver une forte dimension financière aussi bien au niveau de l'incitation qu'au niveau de l'objectif. De ce fait, avec ces méthodes, on peut se demander si la performance sociétale constitue véritablement un but en soi ou s'il s'agit uniquement d'un moyen permettant d'atteindre des objectifs financiers dans l'optique de satisfaire les actionnaires (Germain et Trébucq, 2003). Or, la responsabilité sociétale repose sur la recherche de comportements conformes aux normes, valeurs et attentes de l'ensemble des différentes parties prenantes. Un tel modèle partenarial est, en revanche, au cœur de la théorie des conventions qui se fonde sur la satisfaction d'objectifs multiples et divergents (Gomez, 1994, p. 219). Cette théorie semble donc plus adaptée à la problématique de la responsabilité sociétale et, plus précisément, à l'analyse des mécanismes incitant les individus à agir dans le sens de la performance sociétale. Les systèmes de mesure des performances déterminent en effet un cadre conventionnel, normé non seulement par des mécanismes incitatifs formels mais également par des repères socialement construits, qui indique implicitement les comportements à adopter. 


\section{Orienter les comportements à partir de systèmes de mesure de la performance sociétale : une approche par les conventions}

La présentation des principes de la théorie des conventions (2.1) permet d'appréhender les systèmes de mesure de la performance comme des conventions d'effort qui indiquent le comportement généralement adopté par les membres de l'organisation (2.2). Les modalités d'évolution des conventions d'effort «comptables » vers des conventions « sociétales » sont alors analysées au travers des facteurs de suspicion (2.3) et de cohérence morphologique (2.4.).

\subsection{Définitions et principes « conventionnels »}

L'approche conventionnaliste, qui est perceptible à la fin des années 1980 en France, a pour ambition d'améliorer la compréhension des mécanismes socioéconomiques en étudiant l'élaboration, l'articulation et la signification des règles conventionnelles (Gensse 2003, p. 14). Les tenants de cette théorie mettent en avant que le comportement des individus est influencé par « ce qui se fait » dans l'organisation et non pas seulement à partir de systèmes d'incitations individuelles dans une perspective contractualiste. En effet, «les conventions sont un ensemble de règles prescrivant des comportements dans un contexte déterminé » (Brousseau, 1993) et, plus précisément, un «ensemble de critères, implicites ou explicites auxquels un individu se réfère au moment de décider » (Gomez, 1996, P. 182). Il s'agit de l'ensemble des repères communs qui permettent l'action en désignant les comportements «normaux », c'est- 
à-dire les choix effectués par d'autres individus supposés agir de façon identique. Il suffit donc d'imiter pour donner du sens au choix individuel.

La force de ces conventions semble importante puisqu'elles reposent sur une construction sociale de l'action collective. Plus précisément, pour décider d'agir dans un sens ou dans un autre il suffit aux individus d'observer ce qui se pratique habituellement. De telles pratiques entraînent des économies de savoir et de temps car leur connaissance de la part des agents leur permet de sélectionner le comportement qui correspond le mieux aux circonstances, sans avoir à étudier les caractéristiques des comportements ou encore à négocier une solution avec les autres agents. Le conformisme à la convention permet de sortir de ce blocage. L'existence d'une convention permet à l'individu de réduire son niveau d'incertitude concernant tant le comportement des autres que les conditions et les déterminants de ses propres décisions. L'individu est donc naturellement incité à ne pas changer de convention. Ces développements signifient que les comportements des utilisateurs des systèmes de mesure de la performance sociétale dépendent des conventions d'efforts qu'incarnent ces systèmes.

\subsection{Le système de mesure de la performance : une convention d'effort}

En effet, les systèmes de mesures de la performance constituent des conventions d'effort, c'est-à-dire une structure de coordination des comportements des agents travaillant dans une organisation. De telles conventions établissent le niveau «d'effort communément admis comme normal» (Gomez, 1994, p. 182) puisqu'elles émettent des règles établissant l'implication de l'agent dans le groupe. Il en est ainsi de la comptabilité des coûts qui joue le rôle de convention d'effort dans la mesure où « elle constitue un vaste système métrologique destiné à fournir aux acteurs des repères quantitatifs conventionnels »(Gomez, 1996, p. 249). 
De tels systèmes de mesure permettent donc d'établir l'accord conventionnel sur l'effort selon un objectif d'efficience commun. Ces systèmes d'évaluation permettent «la transmission d'informations sur l'implication attendue et/ou effective »(Gomez, 1994, p. 191) et une relation entre l'effort et les normes de mesure établies sur celui-ci. Les règles de l'effort sont rappelées à ce moment, mais aussi confirmées ou amendées par la réalité de l'implication observée. Les systèmes de mesure de la performance comptable répondent ainsi aux conditions conventionnelles telles que définies par Lewis (1969). Un système de mesure de la performance sociétale ou, en d'autres termes, une convention d'effort sociétale, peut donc se heurter à la convention d'effort du système comptable traditionnellement utilisée dans les organisations. Une des raisons réside dans la représentation partagée de l'effort à fournir basé sur la dimension financière. Toutefois, ce n'est pas parce que les individus adoptent des conventions qu'ils ne disposent d'aucune liberté. En effet, les individus «peuvent tricher, désobéir, tenter de changer le système conventionnel en place, faire référence à des systèmes conventionnels différents... ils peuvent agir contre la convention »(Gomez, 1996, p. 177). Ce type de comportement résulte d'une des conditions conventionnelles qui stipule qu'il existe au moins une autre proposition alternative à la résolution du problème d'incertitude. En d'autres termes, différentes conventions pouvant se concurrencer, l'individu choisit celle à laquelle il souhaite adhérer. L'utilisation d'un système basé sur des critères plus vastes, notamment sociétaux, peut alors remettre en cause la convention d'effort communément acceptée. La problématique est alors de rechercher les modalités d'évolution de la convention d'effort « comptable » vers une convention d'effort «sociétale » liée au système de mesure de la performance. 


\subsection{Les modalités d'évolution de la convention d'effort «comptable» vers une convention d'effort « sociétale »}

Selon les représentants de la théorie des conventions, une telle évolution nécessite une suspicion de convention, c'est-à-dire «une information qui tend à sa remise en cause » (Gomez, 1994, p. 121) et, plus précisément, qui indique que le comportement généralisé n'est pas celui que l'on croyait. Cette suspicion peut naître de manière exogène par des agents extérieurs à la convention qui concourent à la production d'une alternative. Elle peut également apparaître de manière endogène. Ce sont les contradictions dans la morphologie de la convention qui font alors naître des doutes chez les adopteurs.

La théorie des conventions identifie deux facteurs exogènes (Amblard, 2003, p. 143) :

- le contact : lorsque des populations sont amenées à se rapprocher physiquement ou par l'intermédiaire des médias ou des technologies de la communication, des alternatives conventionnelles peuvent apparaître. De nombreux secteurs d'activités s'impliquent dans la responsabilité sociétale. Dans la mesure où les réseaux de responsables d'entreprises et de gestionnaires se développent la convention alternative peut se diffuser dans les secteurs économiques. Ainsi, les nombreuses firmes en relation, par exemple, avec Philips, Suez, Danone, Monoprix ou encore Lafarge qui produisent des tableaux de bord et des systèmes de reporting complet en matière de responsabilité sociale, sont en contact avec les utilisateurs de la convention d'effort du même nom.

- la réglementation publique : les textes publiés sont de nature à réorienter les comportements. La conformité à ces textes dépend de la conviction que ces comportements seront adoptés par toute la population. Concernant les systèmes de mesure de la performance la loi NRE oblige 
les entreprises, depuis le 01 janvier 2002, à délivrer un rapport sociétal et environnemental comportant, en principe, des indicateurs. Cette obligation devrait inciter les entreprises à adopter un comportement allant dans ce sens car, si des contradictions apparaissaient entre ces rapports et les pratiques, leur crédibilité serait remise en doute.

Selon Amblard (2003, p. 143), trois types de facteurs endogènes sont en outre susceptibles de faire émerger une suspicion de convention:

- la dissidence : il s'agit d'un groupe d'individus qui ne se conforme plus à la convention en place. Ces pratiques peuvent avoir un effet d'électrochoc sur l'ensemble de la population qui peut la remettre en doute dans la mesure où elle est informée de l'existence d'une convention alternative et de l'adhésion d'un groupe dissident à celle-ci. Par exemple, l'utilisateur des systèmes de mesure de la performance peut être, dans sa sphère privée, un «consom'acteur » (Pastore-Reiss et Naillon, 2002) qui peut adopter des comportements responsables. Il peut alors pratiquer le boycottage des marques qui ne respectent pas les principes du développement durable et transférer de telles pratiques dans sa sphère professionnelle. Il participe alors, à travers l'entreprise, à un projet collectif citoyen ce qui développe une dissidence.

- la dissonance constitue une inadéquation du discours délivré par la convention face aux transformations contextuelles. Cette dissonance peut jeter un doute sur le respect de la convention en place. Une convention d'effort orientée vers une métrologie comptable est adaptée dans des contextes où les fondements de la compétitivité des entreprises reposent principalement sur les coûts. Lorsque ces fondements se diversifient, la convention peut devenir dissonante si elle n'évolue pas. L'ensemble des débats, réflexions et travaux en contrôle de gestion dans les années 1980 critiquant l'inadaptation de la mesure de la 
performance face aux modifications des stratégies organisationnelles (Amintas, 1999) constitue une illustration de cette dissonance.

- l'intention stratégique : elle repose sur la volonté d'un groupe d'influencer le reste de la population, et plus précisément de renverser la convention en place. La nomination de «Responsables du Développement Durable » par exemple chez AGF, Rhodia ou encore Schneider Electric, la déclaration de politique générale dans le sens de la responsabilité sociétale et la dotation d'instances chargées d'intégrer celle-ci constituent de telles démarches d'intentions stratégiques.

\subsection{La cohérence morphologique de la convention d'effort « sociétale »}

Ces développements constituent donc un essai relatif aux conditions d'émergence d'une convention d'effort liée au système de mesure de la performance sociétale. Toutefois, les tenants de la théorie des conventions considèrent que lorsque deux conventions sont mises en compétition, c'est celle qui donne l'information la plus convaincante qui l'emporte. «Une convention est convaincante lorsqu'elle est cohérente »(Gomez, 1994, p. 123), c'est-à-dire que les éléments de sa morphologie ne sont pas contradictoires ou dissonants entre eux et qu'ils n'émettent pas des informations opposées qui brouilleraient les observations des adopteurs. La morphologie d'une convention comporte deux dimensions.

La première porte sur l'énoncé, c'est-à-dire ce qu'est la convention :

- L'énoncé d'une convention d'effort permet de savoir sur quoi se fonde l'excellence du travail, ce qui est considéré comme bon, positif : le principe commun. Le système de mesure de la performance est explicite sur cet énoncé puisqu'il indique le niveau d'effort à tenir et à évaluer en matière de responsabilité sociétale. 
- L'énoncé porte également sur les différents types d'adopteurs de la convention. La distinction perceptible dans une convention d'effort «sociétale » se traduit par la hiérarchie susceptible de représenter les objectifs communs de l'organisation, les concepteurs des systèmes, et enfin les utilisateurs.

- L'énoncé indique à l'adopteur la sanction d'une non-adhésion à la convention. Pour une entreprise, la sanction d'un refus d'une orientation vers la performance sociétale peut se traduire par des coûts d'opportunité puisqu'une telle orientation semble conduire à une augmentation à long terme des indices boursiers (Férone et al., 2002) et de l'efficacité. Une démarche de respect de l'environnement, par exemple, permet d'accroître la rentabilité par une économie de coût. La Société Générale économiserait ainsi un million d'euros par an grâce à une meilleure utilisation de l'énergie solaire notamment (Steinmann, 2002, p. 32). De même, STMicroelectronics aurait économisé 45 millions de dollars en 2001 grâce à une diminution de la consommation d'énergie (Laville, 2002, p. 166). La décision de non-adoption d'une convention d'effort «sociétale» par un responsable d'unité peut être remise en cause s'il perçoit des différentiels de résultat dans des unités voisines. L'image de l'entreprise peut également souffrir d'une absence d'adhésion à une telle convention face aux investisseurs, à la société civile, ou encore aux agences de notation sociétale.

La seconde dimension de la morphologie d'une convention porte sur la manière dont est véhiculée l'information c'est-à-dire le « dispositif matériel ». Il comporte deux aspects :

- Les contacts entre les adopteurs de la convention permettent de contrôler le phénomène d'imitation des comportements. Les réunions de suivi organisées périodiquement dans le cadre d'un système de mesure de la performance constituent de tels moments de contacts. Ces relations permettent de retracer la performance de l'unité de chaque responsable de service, c'est-à-dire l'efficacité (réalisation des objectifs) et l'efficience (rapport entre les moyens et les 
résultats). Ces réunions permettent aux individus de vérifier le degré d'effort fourni et à fournir par les autres membres, notamment lorsque les discussions portent sur les actions de correction à mettre en œuvre pour réguler d'éventuels écarts.

- La technique utilisée permet, d'une part, d'indiquer le comportement à adopter et, d'autre part, facilite pour l'adopteur l'envoi d'un signal prouvant que son comportement est conforme. Même si le principe de la convention repose sur du mimétisme, il semble tout de même nécessaire de transmettre une information indiquant aux «individus que le comportement généralisé a changé »(Gomez, 1994, p. 121). Le sens des mots, «les nuances sémantiques vont peser lourdement dans la nature du message conventionnel diffusé »(Gomez, 1994, p. 113). Or, cet exercice de communication est délicat dans la mesure ou le langage et la capacité de compréhension ne sont pas les mêmes selon la place que l'on occupe dans l'organisation. En effet, les pratiques « linguistiques » sont multiples dans les organisations et impliquent des acteurs n'appartenant pas aux mêmes communautés langagières (Girin, 1990 ; Mélèse, 1990). En raison de ces limites, une politique de développement durable doit être traduite aux parties prenantes dans les langues « locales ». De ce fait, les individus ayant une intention stratégique de changer la convention d'effort en direction d'une convention « sociétale » peuvent s'impliquer dans des opérations de traduction -au sens de Callon (1999) et Latour (1989)- afin de faciliter le contact entre les conventions et la compréhension de l'énoncé par les autres membres de l'organisation. Ces différences de langage ne constituent, probablement, que la face émergée des difficultés que peuvent rencontrer les organisations souhaitant inciter les individus à fournir des efforts compatibles avec les systèmes de mesure de la performance sociétale. Cette contribution ne constituant qu'un essai, elle ne prétend pas traiter de manière exhaustive de telles difficultés et ne vise qu'à proposer des pistes de réflexion pour l'adhésion à une convention d'effort sociétale. 


\section{Conclusion}

Le développement très net, tant dans la communauté des chercheurs que dans celle des praticiens, de nombreuses réflexions relatives au développement durable et à la responsabilité sociétale des entreprises traduit une évolution de la place et du rôle dévolus aux organisations productives au sein de nos sociétés. Même si, d'un point de vue strictement juridique, les processus de prise de décision et d'exercice du pouvoir au sein des entreprises ne sont pas modifiés, tout laisse à penser que les prochaines années seront placées sous le sceau d'une perméabilité beaucoup plus grande des frontières des organisations aux aspirations du corps social. Dans ce contexte, notre objectif était d'apporter une contribution à l'analyse dynamique des systèmes de mesures des performances.

Nous mobilisons à cet effet l'appareillage de la théorie des conventions afin d'étudier les conditions de modification des conventions d'effort "comptables » en conventions d'effort « sociétales ». Une telle évolution nous semble en effet nécessaire à une modification substantielle des attitudes tant individuelles que collectives. Nous posons enfin la question de la cohérence morphologique de telles conventions et suggérons de recourir à certaines préconisations de la théorie de la traduction afin d'assurer le développement et l'efficacité de ces mécanismes.

Trois ensembles d'arguments, pouvant constituer de fructueuses pistes de recherches ultérieures, nous semblent susceptibles de limiter la portée ou l'opérationnalité de nos préconisations. Tout d'abord, malgré un intérêt scientifique croissant, il reste à établir un lien positif formel entre des choix gestionnaires volontairement orientés «Responsabilité Sociétale » et les performances comptables et financières des organisations. Il s'agira en effet 
probablement de l'argument central déterminant la pérennité et la généralisation -au-delà des grandes entreprises- de la problématique du développement durable. Ensuite, notre analyse repose sur la mobilisation de différents cadres conceptuels permettant d'éclairer certaines conditions de mise en œuvre et de réussite de systèmes renouvelés d'appréciation des performances. Ces cadres conceptuels, et notamment le recours à la théorie des conventions, doivent être largement approfondis. Ainsi, l'environnement des conventions mérite d'être étudié pour analyser les facteurs influençant les individus à choisir leurs ensembles de repères communs. Par ailleurs, la manière dont plusieurs systèmes de mesure de la performance, en tant que conventions d'efforts, s'articulent et interagissent permet d'étudier la dynamique conventionnelle. Enfin, les travaux empiriques portant sur ces systèmes étant rares, il semble important d'analyser leurs évolutions effectives vers l'intégration d'indicateurs de performance sociétale. L'analyse des méthodologies des institutions ou agences de notation sociétale et des outils de contrôle de gestion des organisations peut contribuer à éclairer sensiblement de telles évolutions.

\section{Bibliographie}

Amblard, M. (2003). «Vers une théorie sur la dynamique des conventions », dans M. Amblard (2003), Conventions et management, De Boeck Université, pp. 139-157.

Amintas, A. (1999). « La crise du contrôle de gestion comme crise des savoirs gestionnaires », dans Y. Dupuy (1999). Faire de la recherche en contrôle de gestion, Vuibert, pp. 173-186.

Argyris, C. (1952). The Impact of Budgets on People, Controllership foundation. 
Bergeron, H. (2000). «Les indicateurs de performance en contexte PME, quel modèle appliquer ? », 21e congrès de l'Association Française de Comptabilité, Angers.

Bieker, T. (2002). «Managing corporate sustainability with the Balanced Scorecard : Developing a Balanced Scorecard for Integrity Management », Oikos $\mathrm{PhD}$ summer academy.

Boisvert, H. (1991). Le contrôle de gestion : vers une pratique renouvelée, Editions du Renouveau Pédagogique Inc, Ottawa, Canada.

Bollecker, M. (2001). Systèmes d'information et contrôle des services opérationnels, Thèse de doctorat en Sciences de Gestion, Université Nancy 2.

Brousseau, E. (1993). L'économie des contrats, Presses Universitaires de France.

Carroll, A. (1999). «Corporate Social Responsability - Evolution of a Definitional Construct », Business \& Society, vol. 38(3), september, pp. 268-295.

Callon, M. et al. (1999). Réseau et coordination, Economica.

Capron, M. (1995). «Vers un renouveau de la comptabilité des ressources humaines ? », Revue Française de Gestion, novembre - décembre, pp. 46-54.

Capron, M. (2003). «Un nouvel instrument d'auto-évaluation des organisations : le bilan sociétal », Revue Comptabilité, Contrôle et Audit, mai, pp. 55-70.

Charreaux, G. et P. Desbrières (1998). « Gouvernance des entreprises : valeur partenariale contre valeur actionnariale », Finance Contrôle Stratégie, vol. 1, n² , juin, pp. 5788.

Chauveau, A. et J.-J. Rosé (2003). L'entreprise responsable, Editions d'organisation.

Chiapello, E. et H. Delmond (1994). «Les tableaux de bord, outil d'introduction du changement », Revue française de gestion, janvier-février, pp. 49-58.

Commission des Communautés Européennes (2001). Promouvoir un cadre pour la responsabilité sociale des entreprises, Bruxelles, 35 pages. 
DiMaggio, P. et W. Powell (1991). The new institutionalism in organizational analysis, University of Chicago Press.

Edvinsson L. et M.S. Malone (1999). Le capital immatériel de l'entreprise, Maxima Laurent du Mesnil Editeur.

Férone, G. et al. (2002). Le développement durable : des enjeux stratégiques pour l'entreprise, Les Editions d'Organisation.

Gélinier, O. (2004). «La triple responsabilité de l'entrepreneur d'aujourd'hui » dans J. Igalens (coord.) (2004). tous responsables, Editions d'Organisation, pp. 93-108.

Gensse, P. (2003). «Le renouvellement de la vision de la firme», dans M. Amblard (2003). Conventions et management, De Boeck Université, pp. 13-23.

Germain, C. et S. Trébucq (2003). «L'intégration des dimensions sociétales dans le pilotage de la performance de l'entreprise : problématiques et enjeux », Actes de colloque de l'EFMD, 16-17 octobre, Nantes.

Girin, J. (1990). «Problèmes du langage dans les organisations » dans J.-F. Chanlat (1990). L'individu dans l'organisation. Les dimensions oubliées, Editions Eska.

Gomez, P.Y. (1996), Le gouvernement des entreprises, Interéditions.

Gomez, P.Y. (1994), Qualité et théorie des convention, Economica.

Gray, R. (1992). «Accounting and Environmentalism : An Exploration of the Challenge of Gentily Accounting for Accountability Transparency and Sustainability » Accounting, Organisations and Society, vol. 17, $\mathrm{n}^{\circ}$ 5, pp. 399-425.

Greenwood, R. et C. Hinings (1996). «Understanding radical organizational change : bringing together the old and the new institutionalism », Academy of Management Review, october, vol. 21, n 4 , pp. 1022. 
Hockerts, K. (2001) «Corporate Sustainability Mangement, Towards Controlling Corporate Ecological and Social Sustainability », Proceedings of Greening of Industry Network Conference, 21-24 January, Bangkok.

Holliday, C. et al. (2003), De la parole aux actes, 67 expériences de développement durable dans l'entreprise, Victoires Editions, PUF.

Hopwood, A.G. (1972). «An Empirical Study of the Role of Accounting Data in Performance Evaluation », Journal of Accounting Research, pp. 156-182

Igalens, J. (2003a). Etude des relations entre les entreprises et les organisations de la société civile autour du concept de responsabilité sociale, note du LIRHE $\mathrm{n}^{\circ} 370$, janvier, $21 \mathrm{p}$.

Igalens, J. (2003b). « DRH aujourd'hui, DRS demain ? », Personnel, n 442, septembre, pp. 55-57.

Kaplan, R.S. et D.P. Norton (1998). Le tableau de bord prospectif, Les Editions d'Organisation.

Kaplan R.S. et D.P. Norton (2001). Comment utiliser le tableau de bord prospectif. Pour créer une organisation orientée stratégie, Editions d'organisation.

Latour, B. (1989). La Science en action, La Découverte, Paris.

Laville, E. (2002). L'entreprise verte, Village mondial.

Lewis, D.K. (1969). Convention : A philosophical study, Cambridge, Harvard University Press.

Lorino, P. (1991). Le contrôle de gestion stratégique : la gestion par les activités, Dunod, Paris.

Malo, J.L. (2000). «Tableaux de bord », in B. Colasse (Éds.), Encyclopédie de Comptabilité, Contrôle de Cestion et Audit, Économica. 
Malo, J.-L. et J.-C. Mathé (2000). L'essentiel du contrôle de gestion, Les Editions d'organisation.

Mathieu, P. (2000). «Insaisissable productivité ?», Direction et Gestion, $\mathrm{n}^{\circ} 186$, novembre-décembre, pp. 13-22.

Mavrinac, S. et A.G. Siesfeld (1998). «La délicate mesure de l'immatériel», L'Expansion Management Review, décembre, pp. 31-38.

Mélèse, J. (1990). Approches systémiques des organisations, Les Editions d'Organisation.

Merchant, K.A. (1985). «Budgeting and the Propensity to Create Budgetary Slack» Accounting, Organizations and Society, vol. 10, n 2, pp. 201-210.

Mintzberg, H. (1982). Structure et dynamique des organisations, Les Editions d'organisation.

Oger, B. et D.E. Pratt (2002). «Modèles de création de valeur en Europe et aux EtatsUnis : une comparaison des modèles de l'EFQM et du Baldrige Award Criteria », Revue Comptabilité, Contrôle et Audit, mai, pp. 85-98.

Pastore-Reiss, E. et H. Naillon (2002). Le marketing éthique, Village mondial.

Perez, R. (2003). La gouvernance de l'entreprise, Editions La découverte, collection Repères,

Steinmann, L. (2002). «Une banque responsable», Enjeux Les Echos, Novembre, p. 32.

Stephany, D. (2003). Développement durable et performance de l'entreprise, Editions Liaisons.

Wiedemann-Goiran, T. et al. (2003). Développement durable et gouvernement d'entreprise : un dialogue prometteur, Editions d'organisation, Les cahiers de l'O.R.S.E.. 
World Commission on Environment and Development (1987). Our common future The Brundtland Report, Oxford University Press.

Wood D. (1991). «Corporate social performance revisited», Academy of Management Review, vol. 16(4), pp. 691-718. 